\title{
Intraoperative Neuromonitoring for Thyroid Surgery: The Proven Benefits and Limitations
}

\author{
Sung Yong Choi (ib) $\cdot$ Young-lk Son (i) \\ Department of Otorhinolaryngology-Head and Neck Surgery, Samsung Medical Center, \\ Sungkyunkwan University School of Medicine, Seoul, Korea
}

Incidence of temporary recurrent laryngeal nerve (RLN) palsy ranges from $2 \%$ to $13 \%$ and permanent palsy ranges from $0.4 \%$ to $5.2 \%$ after thyroidectomy [1]. Since Lahey and Hoover [2] revolutionized thyroid surgery by advocating routine identification of the RLN, direct visualization of RLN and capsular dissection has become the gold standard for the prevention of RLN palsy during thyroid surgery. Despite cautious RLN visual identification, RLN nerve injury can still occur because of anatomical variation, surgeon inexperience, and difficult situations including large goiter, revision surgery and invasive malignancy [3]. A major drawback of reliance on visualization alone is the inability of the surgeon to predict the functional integrity of a structurally intact nerve. Possible mechanisms of RLN injury include not only transection but also clamping, ligation, traction, thermal injury, and ischemia [4].

The use of intraoperative neuromonitoring (IONM) during thyroidectomy allows confirming the functional integrity of the RLN as well as facilitates identification of the RLN before visualization during operations, especially for high-risk situations. In this context, monitoring of both evoked and spontaneous electromyographic (EMG) activity of vocalis muscle was expected to substantially reduce the rate of RLN injury. Despite initial enthusiasm for IONM, most studies failed to prove a significant difference in the incidence of RLN injury between IONM and visualization alone $[5,6]$. In contrast, a small number of studies did report significant benefits of IONM in higher-risk thyroidectomy; reoperation, thyroidectomy for invasive malignancy, thyrotoxicosis or retrosternal goiter [7]. Overall, the utility of IONM in reducing the rate of RLN injury is largely unproven for thyroid operations in general, but it may have distinct value in high-risk thyroidectomies.

Despite the lack of conclusive data showing benefit, it would be still helpful and necessary to understand various advantages of IONM as an adjunct tool for thyroid surgery. IONM applica- tion in thyroidectomy can facilitate nerve identification, which is associated with a higher chance of nerve identification and shorter operation time [8]. IONM also informs surgeons of the functional integrity of the RLN, which can aid intraoperative decision making for surgical extent, type of procedure and airway management, especially when bilateral RLN are at risk [9]. Through serial nerve stimulating from distal to proximal direction, the identification of the injured point of RLN may allow the surgeon to recover the damage from clipping or suturing and avoid permanent RLN injury. Additionally, IONM can help identify the external branch of the superior laryngeal nerve and avoiding its injury, which is especially meaningful for professional voice users [10].

Currently, IONM has some technical limitations which surgeons need to recognize. A nerve stimulator is repeatedly used for nerve identification during surgery but the interval between each stimulation goes unsupervised. Lamade et al. [11] proposed a repetitive, short-interval stimulation system, which is called continuous IONM (C-IONM) technology. In C-IONM systems, a vagal nerve was indirectly stimulated by an automatic periodic stimulation electrode, which can allow real-time monitoring of the amplitude and latency of EMG on the RLN. However, the superiority of C-IONM on RLN preservation, compared with conventional IONM, is also still controversial. Furthermore, some studies report that frequent vagal nerve stimulation is associated with cardiac arrhythmias, bronchospasm and hemodynamic alterations [12]. Another problem is the wide range of sensitivity and positive predictive values of the recording part to measure EMG of vocalis muscle. Because EMG measures the electrical phase difference between two electrodes, it can be affected by saliva or sputum in the larynx. Contact between the vocal fold and the electrode can be unstable by the movement or malposition of the EMG endotracheal tube or needle electrodes [13].

Copyright () 2019 by Korean Society of Otorhinolaryngology-Head and Neck Surgery.

This is an open-access article distributed under the terms of the Creative Commons Attribution Non-Commercial License (http://creativecommons.org/licenses/by-nc/4.0)

which permits unrestricted non-commercial use, distribution, and reproduction in any medium, provided the original work is properly cited. 
The benefit of routine IONM during thyroid surgery is still controversial. However, the use of IONM as an adjunct to visualize RLN and to verify the functional integrity can be helpful especially in high-risk situations. Reliable and safe C-IONM methods need to be further developed.

\section{CONFLICT OF INTEREST}

No potential conflict of interest relevant to this article was reported.

\section{ORCID}

Sung Yong Choi https://orcid.org/0000-0002-4865-5677

Young-Ik Son https://orcid.org/0000-0002-2114-8085

\section{REFERENCES}

1. Hayward NJ, Grodski S, Yeung M, Johnson WR, Serpell J. Recurrent laryngeal nerve injury in thyroid surgery: a review.ANZ J Surg. 2013 Jan;83(1-2):15-21.

2. Lahey FH, Hoover WB. Injuries to the recurrent laryngeal nerve in thyroid operations: their management and avoidance.Ann Surg. 1938 Oct;108(4):545-62.

3. Lin HS, Terris DJ. An update on the status of nerve monitoring for thyroid/parathyroid surgery. Curr Opin Oncol. 2017 Jan;29(1):14-9.

4. Snyder SK, Lairmore TC, Hendricks JC, Roberts JW. Elucidating mechanisms of recurrent laryngeal nerve injury during thyroidectomy and parathyroidectomy. J Am Coll Surg. 2008 Jan;206(1):123-30.

5. Cirocchi R,Arezzo A, D’AndreaV,Abraha I, Popivanov GI,Avenia N, et al. Intraoperative neuromonitoring versus visual nerve identification for prevention of recurrent laryngeal nerve injury in adults undergoing thyroid surgery. Cochrane Database Syst Rev 2019;1:CD012483.

6. Mirallie E, Caillard C, Pattou F, Brunaud L, Hamy A, Dahan M, et al. Does intraoperative neuromonitoring of recurrent nerves have an impact on the postoperative palsy rate? Results of a prospective multicenter study. Surgery. 2018 Jan;163(1):124-9.

7. Wong KP, Mak KL, Wong CK, Lang BH. Systematic review and meta-analysis on intra-operative neuro-monitoring in high-risk thyroidectomy. Int J Surg. 2017 Feb;38:21-30.

8. Randolph GW, Dralle H; International Intraoperative Monitoring Study Group, Abdullah H, Barczynski M, Bellantone R, et al. Electrophysiologic recurrent laryngeal nerve monitoring during thyroid and parathyroid surgery: international standards guideline statement. Laryngoscope. 2011 Jan;121 Suppl 1:S1-16.

9. Chiang FY, Lee KW, Chen HC, Chen HY, Lu IC, KuoWR, et al. Standardization of intraoperative neuromonitoring of recurrent laryngeal nerve in thyroid operation. World J Surg. 2010 Feb;34(2):223-9.

10. Hurtado-Lopez LM, Diaz-Hernandez PI, Basurto-Kuba E, ZaldivarRamirez FR, Pulido-Cejudo A. Efficacy of intraoperative neuro-monitoring to localize the external branch of the superior laryngeal nerve. Thyroid. 2016 Jan;26(1):174-8

11. LamadeW, Meyding-Lamade U, Buchhold C, Brauer M, Brandner R, Uttenweiler V, et al. First continuous nerve monitoring in thyroid gland surgery. Chirurg. 2000 May;71(5):551-7.

12. Terris DJ, Chaung K, DukeWS. Continuous vagal nerve monitoring is dangerous and should not routinely be done during thyroid surgery. World J Surg. 2015 Oct;39(10):2471-6.

13. Sung ES, Lee JC, Shin SC, Kwon HK, Na HS, Park DH, et al. Development of a novel intraoperative neuromonitoring system using an accelerometer sensor in thyroid surgery: a porcine model study. Clin Exp Otorhinolaryngol. 2019 Nov;12(4):420-6. 